\title{
Article
}

\section{Design, synthesis of spiro quinazolinone compacted with chitosan through DFT approach for interference the antimicrobial activity}

\author{
Eman Abdel-Nasser ${ }^{1}$, Jehan A. Hafez ${ }^{1}$, Radwa M. Badawy ${ }^{1}$, Hadeer I. Mohamed ${ }^{2}$ and Sameh A. Rizk ${ }^{3, *}$ \\ Department of Entomology, Faculty of Science, Ain Shams University, Cairo 11566, Egypt. \\ Department of Biophysics, Faculty of Science, Ain Shams University, Cairo 11566, Egypt. \\ Department of Chemistry, Faculty of Science, Ain Shams University, Cairo 11566, Egypt. \\ * Correspondence: samehrizk@sci.asu.edu.eg; samehrizk2006@gmail.com; Tel.: +20 01116988669; +20107350672; Fax: \\ +224662917 .
}

Received: 18 November 2019; Accepted: 3 December 2019; Published: 15 December 2019.

\begin{abstract}
In this work, we present a newly three antimicrobial chitosan, 2-benzoxazinyl benzoic acid (BBA) and novel biopolymer of Chitosan-spiroquinazolinone (SQC) via coupling of chitosan with benzoxazinyl benzoic acid, for promoting the antimicrobial activity of inherent chitosan. Modification in the chemical structure of the synthesized product SQC was confirmed using FT-IR and UV analysis. The antimicrobial activities of Chitosan, and BBA compounds were expressively increased more than chitosan-spiroquinazolinone SQC. Minimum inhibitory concentration (MIC) of compound SQC was supposed at $50 \mu \mathrm{g} / \mathrm{ml}$ against tested microorganisms except for B.cereus and C.albicans. The highest concentration of Chitosan would prevent the growth of Gram-+ve upto $99 \%$. However, compound BBA recorded the maximum inhibition percentage versus Gram-+ve approximately $82 \%$. The findings emphasized that the developed Chitosan-Spiroquinazolinone SQC may be blocker for antimicrobial activity to pure chitosan and BBA i.e, stop reaction if possible for actions of antimicrobial treatments.
\end{abstract}

Keywords: Chitosan-spiroquinazolinone (SQC), bio-polymers.

\section{Introduction}

$\mathbf{T}$ he antimicrobial bio-polymers have generally considered as key to the enormous desires for the last years, in decent wound [1], engineer tissue [2], medical textile [3], packaging [4] and water treatment [5]. Mid all natural bio-polymers, chitosan is easily available, found in several insects and microorganisms and considered one of the most effective antimicrobial bio-polymers[6,7]. By simple deacetylation process, chitosan can be easily extracted from chitin via deacetylated unit ( $\beta$-(1-4)-linked d-glucosamine) copolymer as characterized by its basic character and simplified its solubility in the acitic acid, non-toxic, anti-bacterial activity, biodegradability and excellent biocompatibility [8-10]. So, it widely used in the bio-medical applications as a drug carrier [11], antimicrobial [12], antioxidant [13], antitumor [14], and a wound dressing agent $[15,16]$.

In addition, chitosan can be chemically modified for widen its applications via grafting with functionalized monomers [17-22]. As it is well known that chitosan has a decent antimicrobial activity against some types of bacteria, and these activities are interconnected to the quantity of the adsorbed chitosan on the cells of bacteria due to the non-stop mutations of microorganisms coupled with new antimicrobial agents $[23,24]$. Accordingly, scientists are struggling to synthesize novel antimicrobial chitosan derivatives to obstruct the wound infections. Several studies have reported the significant effect of antimicrobial bio-polymers and chitosan itself to enhance the wound healing process through preventing the wound infections that result in tissue maceration [25-27]. It has been reported that chitosan modified diisocyanate (DIMC) [28], O-quaternary ammonium $\mathrm{N}$-acyl thiourea chitosan [29], chitosan-thioglycolic acid [29,30], kanamycin-chitosan nanoparticles [31], crosslinked chitosan [32] and O-amine functionalized chitosan [33] exhibited better antibacterial activity degrees against Escherichia coli, Staphyloccocus aureus and other microorganisms. Likewise, chitosan Schiff 
base derivatives are considered one of the best choices for increasing antimicrobial activity of chitosan, since carbonyl groups of aldehyde or ketone can efficiently couple with $\mathrm{NH}_{2}$ groups of chitosan to form the corresponding chitosan Schiff base with imine characteristic group (-RC=N-) [34]. This leads directly to altering chitosan molecular structure, enhancement its hydrophilicity as well as increasing the positively charged ions, which results in better antibacterial activity compared to the unmodified chitosan.

The present work reports the development of antimicrobial chitosan derivatives that could accelerate the wound healing via hindering the microbial infections [35-37]. Herein, Spiroquinazolinone-chitosan (SQC) was synthesized, characterized and verified using different characterization tools. Furthermore, their antimicrobial activities were examined against various pathogenic microorganisms that frequently irritating infections.

\section{Results and discussion}

\subsection{Chemistry}

It has been synthesized by reaction of anthranilic acid with phthalic anhydride to afford N-benzoyl-anthranilic acid. Refluxing of N-benzoyl-anthranilic acid in acetic anhydride afforded 2-phenyl-4H-3,1-benzoxazin-4-one (1) as outlined in following Scheme [El-Hashash, et.al 2018, 2017]. The reaction of the anthranilic causes the ring opening of the phthalic anhydride to afford the corresponding anthranil intermediate followed by ring closure to the afford the 2-benzoxazinyl-benzoic acid drug.

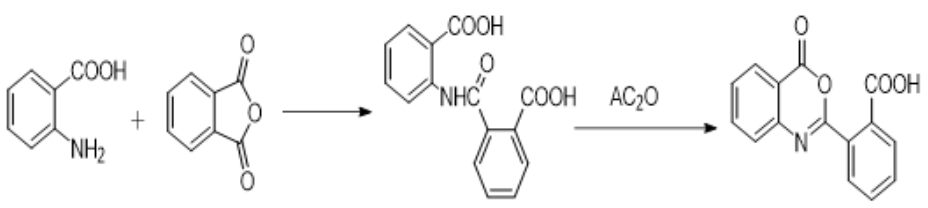

Scheme: outline the formation of the 2-benzoxazinyl-benzoic acid via reaction of the phthalic anhydride with anthranilic acid.

\subsection{The DFT study of the benzoxazinone derivative}

In the same way, DFT simulation of the benzoxazinone deivative outlines the electrophilic sites of the optimazid structure that has LUMO energy $-3.73 \mathrm{eV}$ to interact with the nucleophilic amino group of bacteria and inhibit its enzyme. So, the benzoxazinone is considered good antibacterial reagent. In the same problem occur in which the benzoxazinone drug can scavenge all the active enzyme in the human body and so it must inhibit this drug immidiately after finishing from the harmful bacteria by the chitosn moieties.
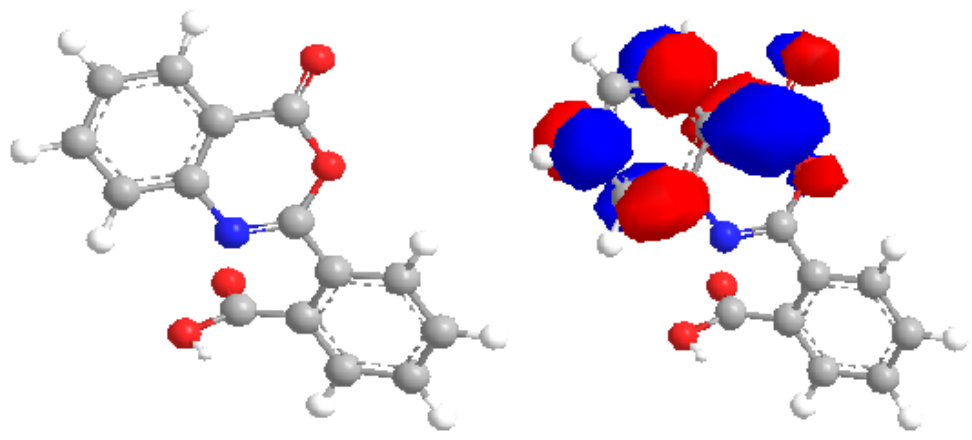

Figure 1. sketch the Optimised structrue of the 2-benzoxazinyl-benzoic acid drug and its LUMO form outline the site of electron density distribution.

\subsection{Inhibition of the drug by chitosan}

Reaction of the amino group of the chitosan with 2-benzoxazinyl benzoic acid afforded the spiro-quinazolinone derivative[ El-Koly A, Rizk S; 2019; El-Hashash M, Rizk S, 2017] as outlined in the 
following Scheme. Formation of spiro benzofuranon-quinazolinone product can confirmed the chitosan has basic character that outlined the following Scheme.

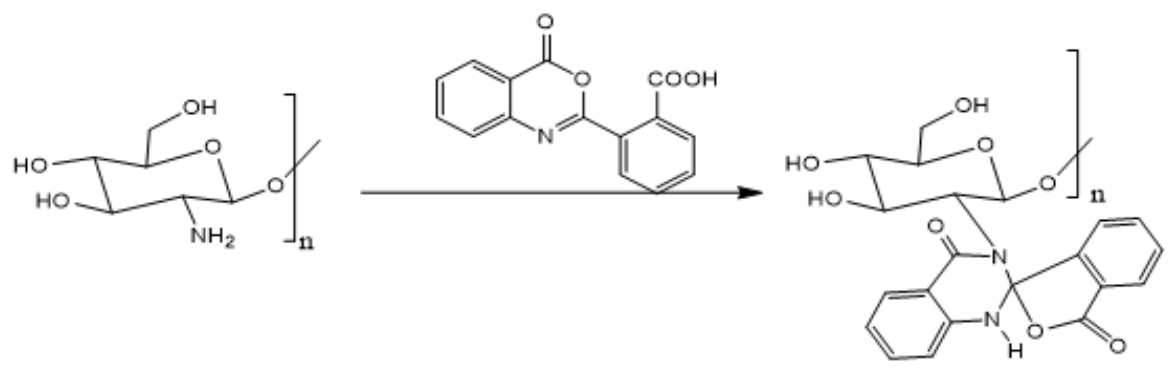

Scheme: outline the formation of the spiro benzofuranone- quinazolinone via reaction of the chitosan with benzoxazin-1-one

DFT simulation still confirmed that HOMO of chitosan is prefered from the HOMO of active enzyme. At this moment, the chitosan is suitable until now to inhibit the synthesized drug. The results of antibacterial and antifungal in the biological part are good agreement with the DFT study of the synthesized organic material.

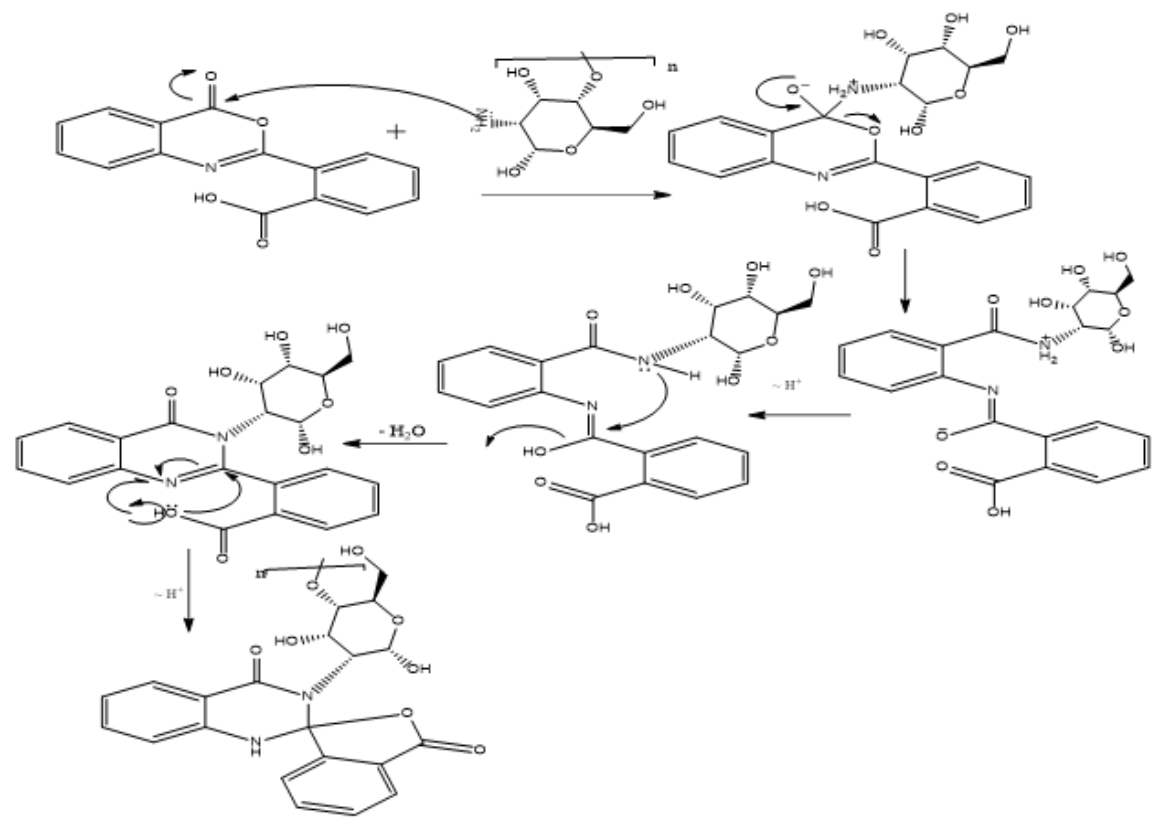

Scheme: outline the mechanistic formation of the spiro drug-chitosan composite via reaction of the phthalic anhydride with anthranilic acid.

Moreover, the chitosan itself has antimicrobial activity but, when it reacts with the drugs, loss the all activity of the chitosan and drug for microbe. The authors concluded that chitosan and drugs alone has become antimicrobial agents but when they mix chimically in one pot promoted ultrasound reaction, become no activity towards the bacteria and fungi.

\subsection{Degree of deacetylation (DD)}

Actually, there are several common methods have been adopted for determination the degree of deacetylation (DD) of chitosan via measuring its free amine groups. Since, it's considered one of the most essential parameters, which directly affect the properties and applications of chitosan. These methods have been conducted by using FT-IR [38], NMR [39], UV-spectrophotometric analysis [40], colloidal titration [40], and potentiometric titration $[41,42]$. The degree of substitution could be estimated via determination the 
content of free amine groups in chitosan before and after reaction. Table 1 represents the calculated values of DD for chitosan and its two Schiff base derivatives.

Table 1. DD and DS values obtained from potentiometric titration method for chitosan and its Spiro derivative.

\begin{tabular}{||ccc||}
\hline Sample & DD $(\%)$ & DS(\%) \\
\hline \hline Chitosan & 93.15 & - \\
Spiro chist quin (SQC) & 81.1 & 12.05 \\
\hline
\end{tabular}

Moreover, the degree of substitution (DS) was $12.05 \%$ for Spiro quinazolinone-Chitosan.

\subsection{FT-IR analysis.}

Figure 2 demonstrates the FT-IR spectral analysis of neat chitosan and its two benzoxazinyl benzoic acid and Spiro chitosan quinazolinone derivative. The spectrum illustrates a typical band of polysaccharides such as common bands between 3200-3400 $\mathrm{cm}^{-1}$ corresponding to hydroxyl and amine groups. 2960 and $1933 \mathrm{~cm}^{-1}$ refer to stretching vibration of $\mathrm{CH}$ and $\mathrm{CH}_{2} .1745$ and $1565 \mathrm{~cm}^{-1}$ assigned to $\mathrm{C}=\mathrm{O}$ spirofuranone with flattened with chitosan. The band at $1370 \mathrm{~cm}^{-1}$ corresponding to $\mathrm{NH} 2$ bend vibration, where multipacks between 1200-1000 $\mathrm{cm}^{1}$ associated with glycoside C-O, C-O-C and C-C bond [21].
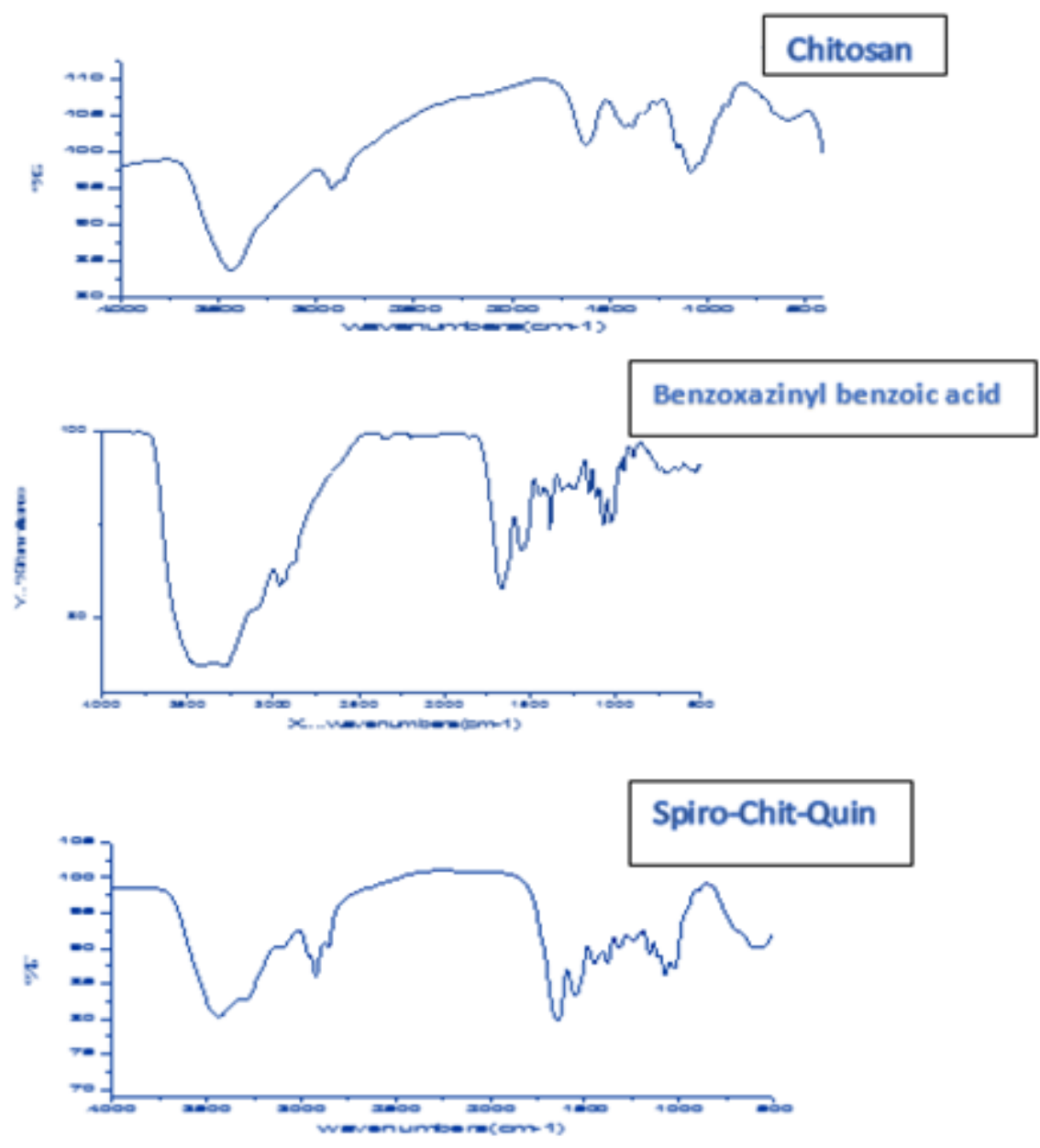

Figure 2. . FT-IR spectra of chitosan, 2-benzoxazinylbenzoic acid drug (BBA) and Spiro quinazolinone chitosan derivative (SQC)

\subsection{Electronic spectra.}

Figure 3 investigates the UV-visible spectra for chitosan, BBA and its novel SQC. It was obvious in case of chitosan that the apparent absorbance band at maximum $230 \mathrm{~nm}$ could be attributed to $n-\sigma^{*}$ transition 
of amine free electrons [43]. Conversely, in case of BBA(I) and SQC(II), the increase in the peak intensity as well as, the observed shift to the higher wavelengths 247 and $361 \mathrm{~nm}$, respectively, could be explained by increasing in donor ability of the substituent to stabilize the excited state [44]. BBA and SQC bonds display a red-shift, which refers to the attachment benzoxazinone and conjugated Spiro precursor respectively to amine groups along chitosan backbone [45]. Furthermore, generation of new bands at higher wavelength from 259.6 to 300nm for BBA (I) and multibands from 361 to $421 \mathrm{~nm}$ for SQC (II) could be ascribed to the formation of spiroquinzolinone furanone, which creates a new transition $n-\sigma^{*}$ at a higher wavelength.

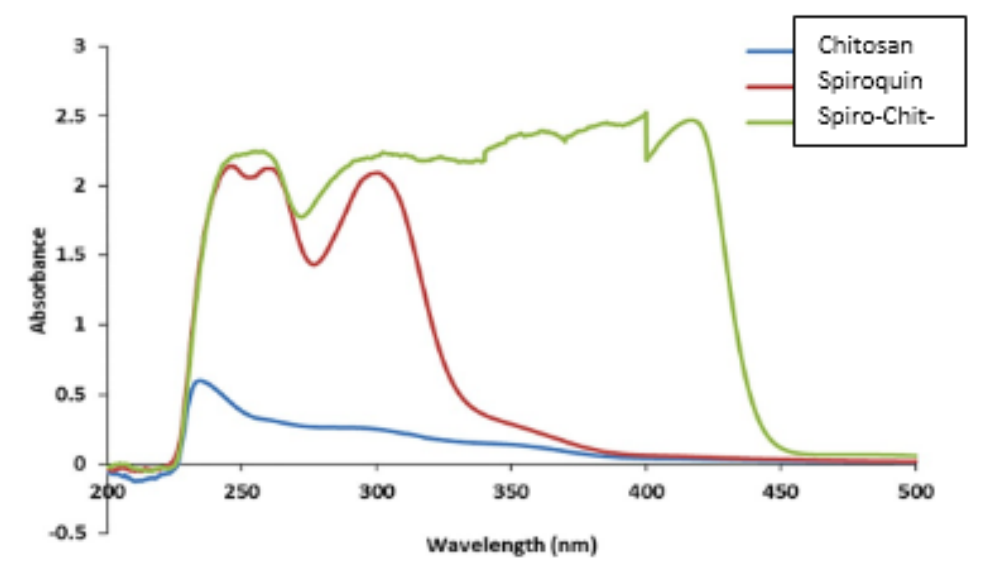

Figure 3. Electronic spectra of chitosan, 2-benzoxazinylbenzoic acid drug (BBA) and Spiro quinazolinone chitosan (SQC)

\section{Antimicrobial assay using Agar-well diffusion method}

Over the recent years, Antimicrobial activities of chitosan and its derivatives have been drawing considerable attention. In addition, mutations of microorganisms to resist the action of antimicrobial materials have enhancing scientists to develop new antimicrobial materials have potential activity against the new version of pathogenic microorganisms. Antimicrobial activities of chitosan, BBA and the developed SQC were initially determined using agar-well diffusion technique against representative Gram-positive bacteria (S. aureus and B. cereus) and Gram-negative bacteria (E. coli, P. aeruginosa, and Salmonella sp.) in addition to yeast strain. For bacterial strains, the activity of chitosan and its derivatives were compared with erythromycin as reference antibiotics, while, nystatin was considered the positive control for yeast strain. The inhibition zones of antimicrobial activities were measured as given in Table 2.

Although the results showed that P. aeruginosa, S. aureus and B. cereus were resistant to the erythromycin, chitosan and its derivatives presented remarkable activities against these tested bacteria. The results exhibited the significant efficacy of the two chitosan and BBA against the indicator microorganisms more than the SQC biopolymer. We could deduce from these findings that BBA (I) demonstrated the highest activity in the presence of all examined microorganisms regardless their structures. The antimicrobial action of chitosan might be differed according to several intrinsic factors including chitosan source, the molecular weight that influences the penetration inside microorganisms, and the synthesis of new chitosan derivatives with novel characteristics that usually diminish the antimicrobial action of chitosan. Three principal mechanisms have been posited to elucidate chitosan interaction with various kinds of microorganisms, which vary based on the cell wall structure and metabolic process [46]. First mechanism is chemical interaction via electrostatic manner between $+v e$ charge of amine groups $(\mathrm{NH} 3+)$ of chitosan with -ve charges on the cell wall of various microorganisms. 2nd mechanism shows the chitosan Mwt which controls its diffusion into the nuclei of microorganisms and binding with DNA to prevent the protein synthesis. 3rd mechanism depends on the bulk chelating of chitosan to metal ions which are vital constituents for microbial growth and metabolic pathways such as spore formation in Gram - + ve bacteria. Accordingly, we propose that the previous mechanisms could be donated together to implement the antimicrobial potency of the synthesized chitosan derivatives.

Earlier studies have reported the BBA compound income its antimicrobial activity, and the newly derivatives showed excellent antibacterial, antifungal, antiparasitic and anticancer activities [47,48]. This 
illustrates the synergistic boost of chitosan activities against the examined microorganisms compared to the synergistic effect of Spiroquinazolinone furanone moiety to the prime chitosan. Significance surprising of these results, MIC, bactericidal and fungicidal activities of the novel chitosan SQC derivative were reduced. The authors can be explain combination of the BBA with chitosan amalgamation that antimicrobial activities in both as blocker for each other to decrease the active sites $\left(\mathrm{NH}_{2}\right)$ lactonic group and $\mathrm{COOH}$ by forming spiroquinazolinone-furanone of chitosan product SQC.

Table 2. Inhibition indices of chitosan and chitosan Schiff bases (I \& II) against E. coli, P. aeruginosa, Salmonella sp., S. aureus, B. cereus, and C. albicans. Values are expressed as mean $\pm \operatorname{SD}(n=3)$.

\begin{tabular}{||ccccc||}
\hline Bacteria & Erythromycin & Chitosan & BBA & SQC \\
\hline \hline E. coli & $11 \pm 0.2$ & $11.5 \pm 0.19$ & $17.7 \pm 0.16$ & $3.7 \pm 0.03$ \\
P. aeruginosa & -ve & $12.6 \pm 0.13$ & $17.2 \pm 0.24$ & -ve \\
Salmonella sp. & $12.5 \pm 0.17$ & $13 \pm 0.17$ & $17.1 \pm 0.15$ & $2.7 \pm 0.04$ \\
S. aureus & -ve & $13.9 \pm 0.19$ & $18.9 \pm 0.21$ & - -ve \\
B. cereus & -ve & $13.6 \pm 0.16$ & $18.1 \pm 0.2$ & -ve \\
Fungi & Nystatin & - & - & - \\
C. albicans & $15.2 \pm 0.22$ & $11.4 \pm 0.14$ & $15.8 \pm 0.25$ & $3.5 \pm 0.18$ \\
\hline
\end{tabular}

\section{Determination of MIC}

In the current research, minimum inhibitory concentrations (MICs) could be defined as the lowest concentrations of chitosan, BBA and SQC that prevent the growth of the tested strains after overnight incubation as presented in Figures [4-6]. These completely hindrance of microbial growth have not attained using the unmodified chitosan and modified SQC (II). With regard to SQC, MIC value was observed at concentration of $50 \mathrm{tg} / \mathrm{ml}$ for all examined microorganisms excluding B. cereus and C. albicans that required $300 \mu \mathrm{g} / \mathrm{ml}$ for inhibiting their growth. The overall results of Spiro chitosan quinazolinone SQC revealed lower activity than the original chitosan; therefore, amino group motivated the antimicrobial activities of chitosan. Moreover, we derived that BBA has the strongest activities against whole indicator microorganisms and this action could be elucidated via boosting the chitosan behavior that clearly showed a synergistic influence on chitosan. This renders SQC the most acceptable candidate for further investigations.

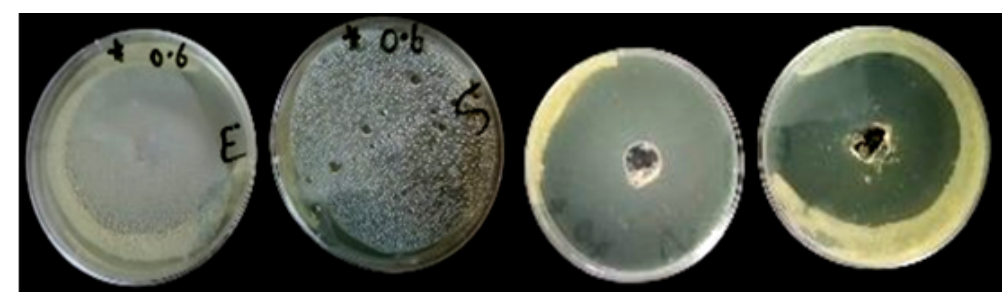

Figure 4. Antibacterial of chitosan and 2-benzoxazinylbenzoic acid

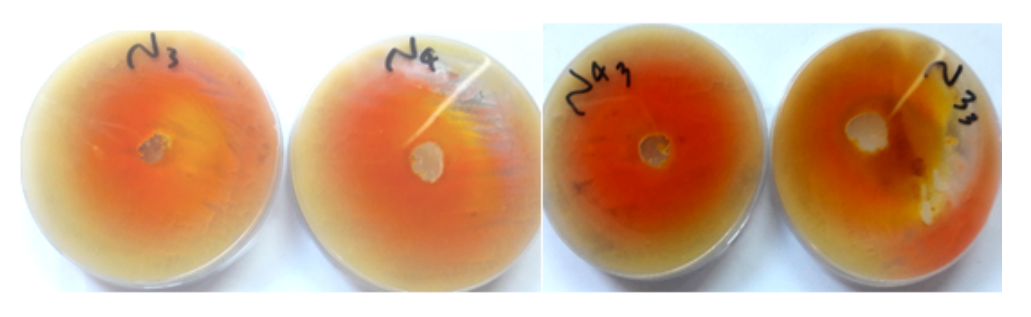

Figure 5. Antifungal of chitosan and 2-benzoxazinyl benzoic acid 


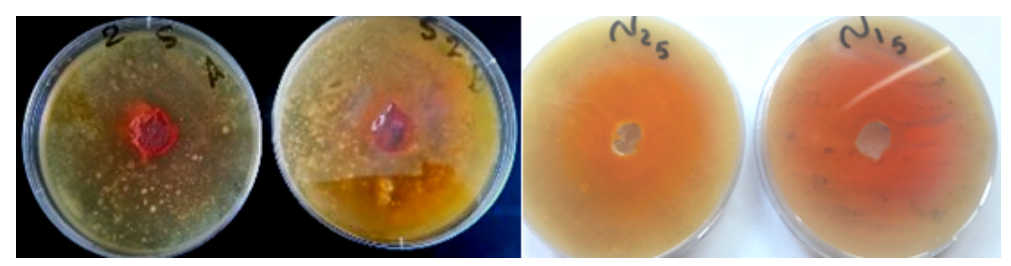

Figure 6. Outline antibacterial and antifungal of Spiroquinazolinone chitosan biopolymer (SQC)

\section{Material and Methods}

\subsection{Materials}

Chitosan (MW. 100,000-300,000 Dalton) was obtained from Across Organics. (New Jersey, USA), 2-benzoxazinylbenzoic acid, (purity 97\%; M.W. 267.15) was acquired from Sigma Aldrich (Germany). Sodium hydroxide pellets (purity $99-100 \%$ ), Sulfuric acid (98\%), Ethanol (99.9\%) were supplied from International co for Supp \& Med. Industries, (Egypt).

\subsection{Microorganisms}

Representative Gram-negative bacteria [Escherichia coli (E. coli.), Pseudomonas aeruginosa (P. aeruginosa) and Salmonella sp.] and Gram-positive [Staphylococcus aureus (S. aureus), and Bacillus cereus (B. cereus)] bacteria in addition to Candida albicans (C. albicans) were utilized to study the antibacterial and antifungal performance of the development materials. All strains were revived from glycerol stocks via growing overnight at $37^{\circ} \mathrm{C}$ and $150 \mathrm{rpm}$ in LB broth medium containing (peptone $1 \%$, yeast extract $0.5 \%$, $\mathrm{NaCl1} \%)$.

\subsection{Synthesis of 2-(4-oxo-4H-benzo[d][1,3] oxazin-2-yl)benzoic acid}

A mixture of $2 \mathrm{~g}$ anthranilic acid $(0.002 \mathrm{~mol})$ and $1.8 \mathrm{~g}$ phthalic anhydride $(0.002 \mathrm{~mol})$ in $20 \mathrm{ml} \mathrm{n}$-butanol was refluxed for $3 \mathrm{~h}$. After cooling the obtained solid was collected, dried and recrystallized from n-butanol to give 1.61g 2-(3-carboxybezamido)-5-benzoic acid in yield 76\% as deep brown crystals: m.p. $180-182^{\circ} \mathrm{C}$. A solution of $2 \mathrm{~g}$ 2-(3-carboxy-bezamido)-5-benzoic acid $(0.01 \mathrm{~mol})$ in $15 \mathrm{ml}$ acetic anhydride was refluxed for $1 \mathrm{~h}$, after cooling the obtained solid was filtered off and crystalized from ethanol to give 1.72 $\mathrm{g}$ of 2-benzoxazinyl-benzoic acid in 75\% yield as white crystals: m.p. $148-150^{\circ} \mathrm{C}$; IR (KBr)v': 3443(broad), 1757, 1707, $1607 \mathrm{~cm}-1 ; 1 \mathrm{H} \mathrm{NMR}$ (CDCl3, $300 \mathrm{MHz}) \delta: 7.2-8.8(\mathrm{ArH}), 4.9$ (s, 1H, OH exchangeable with D2O ); MS (70eV) m/z (100\%): 392.95 (100.0\%), 393.95 (16.2\%), 394.96 (1.2\%). Calculated Elemental Analysis for C15H9NO4 (MW 267): C, 45.83; H, $2.05 ; \mathrm{N}, 3.56$; found $\mathrm{C}, 45.79 ; \mathrm{H}, 2.32 ; \mathrm{N}, 3.46$.

\subsection{General Procedure for the preparation of the chitosan-spiroquinazolone}

An equimolar mixture of 2-benzoxazinyl-benzoic acid (3.03 g $0.01 \mathrm{~mol})$ and chitosan (0.01 mol) in $50 \mathrm{~mL}$ boiling ethanol. The reaction mixture was refluxed for $3 \mathrm{~h}$. The solid that separated after cool was filtered off, washed by petroleum ether (b.p $40-60^{\circ} \mathrm{C}$ ), dried and then, crystallized from DMF. Yield 70\%. m.p. 300-302. IR(KBr)v 3333, 3225(NH), 3056 (CHAr), 1804, 1670 (CO) of spirofuranone and quinazolinone. 1HNMR (DMSO): $\delta$ multiplet at 7.23-7.72 assigned for 8 ArH aromatic protons, singlet peaks at 5,54 and 6.23 a acidic NH and $\mathrm{OH}$ a protons which exchanged in $\mathrm{D} 2 \mathrm{O}$.

\subsection{Physicochemical characterization}

The changes in the chemical structures and surface morphologies of the prepared chitosan, BBA and SQC were investigated by Fourier Transform Infrared Spectrophotometer (FT-IR; Model 8400 S, Shimadzu, Japan) was examined. Moreover, the electronic spectra of the developed chitosan Schiff bases were identified via UV-Vis Spectrophotometer (Ultrospec 2000 Pharmacia BiotechCo., Cambridge, England) in scanning ranged using $0.2 \%$ chitosan or its new Spiroquinazolinone derivatives. The electronic absorbance was investigated in the scanning range 200-500 $\mathrm{nm}$ [43]. 


\subsection{Antimicrobial assay using Agar-well diffusion method}

Agar-well diffusion approach was adopted to assess the antimicrobial activities of pure chitosan, BBA and chitosan SQC derivative against various pathogenic microorganisms (E. coli, P. aeruginosa, Salmonella sp., S. aureus, B. cereus and C. albicans) as previously described. Old overnight cultures of the tested microorganisms were diluted 10-fold in LB broth free medium and their turbidities were adjusted to be equal the McFarland 0.5 standard via measuring at $625 \mathrm{~nm}$, where bacterial strains were $(1-2 \times 108) \mathrm{CFU} / \mathrm{ml}$, while C. albicans was $(1-5 \times 108) \mathrm{CFU} / \mathrm{ml}$. Then, $50 \mu \mathrm{l}$ of the cell suspensions were spread over the surface of LB agar plates using glass spreader. The Agar plates were bored with a metal cork borer to produce wells of $6 \mathrm{~mm}$ in diameter, and $50 \mu l$ of the examined biopolymers were loaded. Afterwards, the Petri dishes were kept in the fridge for $2 \mathrm{~h}$ to diffuse the materials into the agar.

The plates were aerobically maintained at $37^{\circ} \mathrm{C}$ for $18 \mathrm{~h}$, and the antimicrobial activities were then determined via estimating the inhibition zone of microbial growth. Our previous report proved the efficiency of chitosan to inhibit the growth of several microorganisms; therefore, it considers positive control. Moreover, reference antibiotics including erythromycin $15 \mu \mathrm{g} / \mathrm{ml}$ and nystatin (100 units) discs were applied for bacteria and Candida experiments, respectively. This assay was adopted as a screening approach to determine whether the prepared BBA and SQC products have siginficant inhibition against the indicator microorganisms to apply the further studies or the further should be neglected. Therefore, this antimicrobial evaluation for the investigated biopolymer was implemented in triplicate.

\subsection{Determination of minimum inhibitory concentration (MIC).}

Microtiter plate method is one of the most effective approaches for determining the MIC for various antimicrobial agents. Consequently, it was conducted to investigate the impact of different concentrations of chitosan and the new derivatives on the growth of the indicator microorganisms as previously demonstrated. The tested biopolymers were sterilized by $0.22 \mu \mathrm{m}$ syringe filter previous to use. The overnight bacterial cultures were diluted 100-folds in LB broth free medium to optical densities of 0.9 for all microorganisms via measuring the bacterial turbidity at $600 \mathrm{~nm}$. Afterward, $20 \mu 1$ of the bacterial culture suspensions were inoculated into a sterile 96-well microplate, and various levels of filtered native chitosan and modified chitosan $(25,50,100,150,200$ and $250 \mu \mathrm{g} / \mathrm{ml})$ were incorporated. The wells were then completed with LB broth free up to $200 \mu 1$ followed by mixing well employing a bench shaker for $2 \mathrm{~min}$ at $100 \mathrm{rpm}$ and incubated aerobically at $37^{\circ} \mathrm{C}$ for $24 \mathrm{~h}$.

\section{Conclusion}

Chitosan with different function groups (i.e.; hydroxyl and amine groups) has a promising structure to prepare new derivatives. Here, Carbonyl groups of the compound BBA and was coupled with chitosan amine group to produce SQC respectively. The formation of compound SQC was confirmed by monitoring modifications in the chemical structure using spectroscopic analysis, FT-IR, abd elemental analysis. The findings of the antimicrobial activities revealed that antimicrobial activities of chitosan and BBA were significantly boosted by amino and lactonic groups respectively. Furthermore, the cellular toxicity assay pointed to the safety of SQC. The present findings lead us to further this study via preparing a membrane of SQC for investigating in vivo as a promising wound dressing candidate to promote the wounds and burns healing process.

Author Contributions: All authors contributed equally to the writing of this paper. All authors read and approved the final manuscript.

Conflicts of Interest: “The authors declare no conflict of interest."

\section{References}

[1] Catalina, M., Cot, J., Borras, M., Lapuente, J., González, J., Balu, A., \& Luque, R. (2013). From waste to healing biopolymers: biomedical applications of bio-collagenic materials extracted from industrial leather residues in wound healing. Materials, 6(5), 1599-1607.

[2] Croisier, F., \& Jérome, C. (2013). Chitosan-based biomaterials for tissue engineering. European polymer journal, 49(4), 780-792. 
[3] Abdel-Mohsen, A. M., Abdel-Rahman, R. M., Hrdina, R., Imramovský, A., Burgert, L., \& Aly, A. S. (2012). Antibacterial cotton fabrics treated with coreÜshell nanoparticles. International journal of biological macromolecules, 50(5), 1245-1253.

[4] Wu, S. (2014). Effect of chitosan-based edible coating on preservation of white shrimp during partially frozen storage. International journal of biological macromolecules, 65, 325-328.

[5] El-Sayed, E. M., Tamer, T. M., Omer, A. M., \& Mohy Eldin, M. S. (2016). Development of novel chitosan schiff base derivatives for cationic dye removal: methyl orange model. Desalination and Water Treatment, 57(47), 22632-22645.

[6] Benhabiles, M. S., Salah, R., Lounici, H., Drouiche, N., Goosen, M. F. A., \& Mameri, N. (2012). Antibacterial activity of chitin, chitosan and its oligomers prepared from shrimp shell waste. Food hydrocolloids, 29(1), 48-56.

[7] Goy, R. C., Morais, S. T., \& Assis, O. B. (2016). Evaluation of the antimicrobial activity of chitosan and its quaternized derivative on E. coli and S. aureus growth. Revista Brasileira de Farmacognosia, 26(1), 122-127.

[8] Rinaudo, M. (2006). Chitin and chitosan: properties and applications. Progress in polymer science, 31(7), 603-632.

[9] Mi, F. L., Tan, Y. C., Liang, H. F., \& Sung, H. W. (2002). In vivo biocompatibility and degradability of a novel injectable-chitosan-based implant. Biomaterials, 23(1), 181-191.

[10] Tamer, T. M., Valachová, K., Hassan, M. A., Omer, A. M., El-Shafeey, M., Eldin, M. S. M., \& Soltés, L. (2018). Chitosan/hyaluronan/edaravone membranes for anti-inflammatory wound dressing: in vitro and in vivo evaluation studies. Materials Science and Engineering: C, 90, 227-235.

[11] Omer, A. M., Tamer, T. M., Hassan, M. A., Rychter, P., Eldin, M. M., \& Koseva, N. (2016). Development of amphoteric alginate/aminated chitosan coated microbeads for oral protein delivery. International journal of biological macromolecules, 92, 362-370.

[12] Yildirim-Aksoy, M., \& Beck, B. H. (2017). Antimicrobial activity of chitosan and a chitosan oligomer against bacterial pathogens of warmwater fish. Journal of applied microbiology, 122(6), 1570-1578.

[13] Valachová, K., Tamer, T. M., Eldin, M. M.,\& Soltés, L. (2016). Radical-scavenging activity of glutathione, chitin derivatives and their combination. Chemical Papers, 70(6), 820-827.

[14] Xie, F., Ding, R. L., He, W. F., Liu, Z. J. L., Fu, S. Z., Wu, J. B., \& Wen, Q. L. (2017). In vivo antitumor effect of endostatin-loaded chitosan nanoparticles combined with paclitaxel on Lewis lung carcinoma. Drug delivery, 24(1), 1410-1418.

[15] Archana, D., Dutta, J., \& Dutta, P. K. (2013). Evaluation of chitosan nano dressing for wound healing: Characterization, in vitro and in vivo studies. International journal of biological macromolecules, 57, 193-203.

[16] Tamer, T., Collins, M., Valachová, K., Hassan, M., Omer, A., Mohy-Eldin, M., \& Albadarin, A. (2018). MitoQ loaded chitosan-hyaluronan composite membranes for wound healing. Materials, 11(4), 569-73.

[17] Rwei, S. P., \& Lien, C. C. (2014). Synthesis and viscoelastic characterization of sulfonated chitosan solutions. Colloid and Polymer Science, 292(4), 785-795.

[18] Afshar, H. A., \& Ghaee, A. (2016). Preparation of aminated chitosan/alginate scaffold containing halloysite nanotubes with improved cell attachment. Carbohydrate polymers, 151, 1120-1131.

[19] Bukzem, A. L., Signini, R., dos Santos, D. M., Láo, L. M., \& Ascheri, D. P. R. (2016). Optimization of carboxymethyl chitosan synthesis using response surface methodology and desirability function. International journal of biological macromolecules, 85, 615-624.

[20] Jayakumar, R., Prabaharan, M., Reis, R. L., \& Mano, J. (2005). Graft copolymerized chitosanŮpresent status and applications. Carbohydrate Polymers, 62(2), 142-158.

[21] Luo, Y., \& Wang, Q. (2014). Recent development of chitosan-based polyelectrolyte complexes with natural polysaccharides for drug delivery. International journal of biological macromolecules, 64, 353-367.

[22] Raafat, D., \& Sahl, H. G. (2009). Chitosan and its antimicrobial potentialŰ́a critical literature survey. Microbial biotechnology, 2(2), 186-201.

[23] Li, J., Wu, Y., \& Zhao, L. (2016). Antibacterial activity and mechanism of chitosan with ultra high molecular weight. Carbohydrate polymers, 148, 200-205.

[24] Kong, M., Chen, X. G., Xing, K., \& Park, H. J. (2010). Antimicrobial properties of chitosan and mode of action: a state of the art review. International journal of food microbiology, 144(1), 51-63.

[25] Kumar, S., Deepak, V., Kumari, M., \& Dutta, P. K. (2016). Antibacterial activity of diisocyanate-modified chitosan for biomedical applications. International journal of biological macromolecules, 84, 349-353.

[26] Li, Z., Yang, F., \& Yang, R. (2015). Synthesis and characterization of chitosan derivatives with dual-antibacterial functional groups. International journal of biological macromolecules, 75, 378-387.

[27] Croce, M., Conti, S., Maake, C.,\& Patzke, G. R. (2016). Synthesis and screening of N-acyl thiolated chitosans for antibacterial applications. Carbohydrate polymers, 151, 1184-1192. 
[28] Kumar, G. V., Su, C. H., \& Velusamy, P. (2016). Preparation and characterization of kanamycin-chitosan nanoparticles to improve the efficacy of antibacterial activity against nosocomial pathogens. Journal of the Taiwan Institute of Chemical Engineers, 65, 574-583.

[29] Mohamed, N. A., \& Fahmy, M. M. (2012). Synthesis and antimicrobial activity of some novel cross-linked chitosan hydrogels. International journal of molecular sciences, 13(9), 11194-11209.

[30] Tamer, T. M., Hassan, M. A., Omer, A. M., Valachová, K., Eldin, M. S. M., Collins, M. N., \& Soltés, L. (2017). Antibacterial and antioxidative activity of O-amine functionalized chitosan. Carbohydrate polymers, 169, 441-450.

[31] Tamer, T. M., Hassan, M. A., Omer, A. M., Baset, W. M., Hassan, M. E., El-Shafeey, M. E., \& Eldin, M. S. M. (2016). Synthesis, characterization and antimicrobial evaluation of two aromatic chitosan Schiff base derivatives. Process Biochemistry, 51(10), 1721-1730.

[32] Yin, X., Chen, J., Yuan, W., Lin, Q., Ji, L., \& Liu, F. (2012). Preparation and antibacterial activity of Schiff bases from O-carboxymethyl chitosan and para-substituted benzaldehydes. Polymer bulletin, 68(5), 1215-1226.

[33] Kumar, S., Kumari, M., Dutta, P. K., \& Koh, J. (2014). Chitosan biopolymer Schiff base: Preparation, characterization, optical, and antibacterial activity. International Journal of Polymeric Materials and Polymeric Biomaterials, 63(4), $173-177$.

[34] Dong, Y., Xu, C., Wang, J., Wang, M., Wu, Y., \& Ruan, Y. (2001). Determination of degree of substitution for N-acylated chitosan using IR spectra. Science in China Series B: Chemistry, 44(2), 216-224.

[35] Hirai, A., Odani, H., \& Nakajima, A. (1991). Determination of degree of deacetylation of chitosan by 1 H NMR spectroscopy. Polymer Bulletin, 26(1), 87-94.

[36] Tan, S. C., Khor, E., Tan, T. K., \& Wong, S. M. (1998). The degree of deacetylation of chitosan: advocating the first derivative UV-spectrophotometry method of determination. Talanta, 45(4), 713-719.

[37] Kasaai, M. R. (2009). Various methods for determination of the degree of N-acetylation of chitin and chitosan: a review. Journal of agricultural and food chemistry, 57(5), 1667-1676.

[38] Kumar, S., Nigam, N., Ghosh, T., Dutta, P. K., Yadav, R. S., \& Pandey, A. C. (2010). Preparation, characterization, and optical properties of a chitosanÜanthraldehyde crosslinkable film. Journal of applied polymer science, 115(5), $3056-3062$.

[39] Soliman, E. A., El-Kousy, S. M., Abd-Elbary, H. M., \& Abou-zeid, A. R. (2013). Low molecular weight chitosan-based schiff bases: synthesis, characterization and antibacterial activity. American Journal of Food Technology, 8(1), 17-30.

[40] Kumar, S., \& Koh, J. (2013). Synthesis, physiochemical and optical properties of chitosan based dye containing naphthalimide group. Carbohydrate polymers, 94(1), 221-228.

[41] Rahmani, S., Mohammadi, Z., Amini, M., Isaei, E., Taheritarigh, S., Tehrani, N. R., \& Tehrani, M. R. (2016). Methylated 4-N, N dimethyl aminobenzyl N, O carboxymethyl chitosan as a new chitosan derivative: Synthesis, characterization, cytotoxicity and antibacterial activity. Carbohydrate polymers, 149, 131-139.

[42] Abdel-Rahman, R. M., Abdel-Mohsen, A. M., Hrdina, R., Burgert, L., Fohlerova, Z., Pavlinák, D., \& Jancar, J. (2016). Wound dressing based on chitosan/hyaluronan/nonwoven fabrics: Preparation, characterization and medical applications. International journal of biological macromolecules, 89, 725-736.

[43] Duarte, M. L., Ferreira, M. C., Marvao, M. R., \& Rocha, J. (2002). An optimised method to determine the degree of acetylation of chitin and chitosan by FTIR spectroscopy. International Journal of Biological Macromolecules, 31(1-3), 1-8.

[44] Rufián-Henares, J. A., \& Morales, F. J. (2008). Microtiter plate-based assay for screening antimicrobial activity of melanoidins against E. coli and S. aureus. Food Chemistry, 111(4), 1069-1074.

[45] Elkholy, A. E., Rizk, S. A., \& Rashad, A. M. (2019). Enhancing lubricating oil properties using novel quinazolinone derivatives: DFT study and molecular dynamics simulation. Journal of Molecular Structure, 1175, 788-796.

[46] El-Hashash, M. A., Rizk, S. A., \& El-Badawy, A. A. (2018). Ultrasonic Aptitude of Regioselective Reaction of 6-bromo-spiro-3, 1-benzoxazinone2, 1'-isobenzofuran-3', 4-dione Towards Some Electrophilic and Nucleophilic Reagents. Journal of Heterocyclic Chemistry, 55(9), 2090-2098.

[47] El-Hashash, M. A., Rizk, S. A., El-Naggar, A. M., \& El-Bana, M. G. (2017). Regiospecific Isomerization of 2-Benzoxazinon-2-yl Benzoic Acid Toward Some Nitrogen Nucleophiles as Environmental Insecticide. Journal of Heterocyclic Chemistry, 54(6), 3716-3724.

[48] Hussein, M. A., Zyaan, O. H., Abo-bakr, H., Sameh, A., Rizk, S. M. F., Hafez, S. E., \& Omnia, M. (2018). Synthesis, molecular docking and insecticidal activity evaluation of chromones of date palm pits extract against Culex pipiens (Diptera: Culicidae). Inter. J.Mosquito Res. 5(4): 22-32.

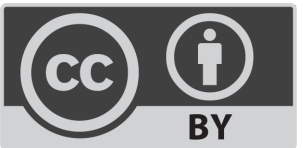

(C) 2019 by the authors; licensee PSRP, Lahore, Pakistan. This article is an open access article distributed under the terms and conditions of the Creative Commons Attribution (CC-BY) license (http://creativecommons.org/licenses/by/4.0/). 